\title{
Granatapfel gegen Prostatakarzinom: Wirkung bestätigt
}

- Auf dem Kongress der American Society of Clinical Oncology (ASCO) im Juni wurde die Wirksamkeit von Granatapfel-Antioxidanzien bei Prostatakrebs durch die Ergebnisse einer aktuellen Studie erneut belegt. In der doppelblinden, randomisierten Studie wurde 104 Prostatakrebs-Patienten mit PSA-Rezidiv nach erfolgloser Primärtherapie Granatapfel-Extrakt verabreicht und der PSA-Verdopplungszeitraum beobachtet. Die Studienteilnehmer waren im Schnitt 74,5 Jahren alt und hatten ein Prostatakarzinom von mittlerer Aggressivität (Gleason-Score 7). Durch die tägliche Aufnahme von Granatapfel-Extrakt über sechs Monate verlängerte sich die mittlere PSAVerdopplungszeit von 11,9 auf 18,5 Monate. Bei $43 \%$ der Studienteilnehmer konnte diese Zeitspanne im Vergleich zum Ausgangswert zu Studienbeginn sogar verdoppelt werden. Bei $13 \%$ wurden fallende PSA-Werte festge- stellt. Signifikante Veränderungen der Testosteronwerte gab es nicht. Zwischen der Einnahme des Granatapfel-Extrakts mit täglich 600 mg Polyphenolen als Gallussäure-Äquivalent und der dreifachen Menge ergab sich kein signifikanter Unterschied in der Wirkung. Bereits 2009 erzielten Pantuck et al. in einer einarmigen Studie eine Verlängerung der PSAVerdopplungszeit von durchschnittlich 15,4 auf 60 Monate, nachdem die Teilnehmer täglich 570 mg Granatapfel-Polyphenole (Saft aus Konzentrat) eingenommen hatten. Präklinische Studien weisen auf eine verlangsamte Entwicklung der Hormonrefraktarität unter Hormonablation hin. Etwa 250 präklinische und 15 klinische Studien bestätigen die günstigen Effekte von Granatapfel-Polyphenolen. Am besten sind die Wirkungen gegen HerzKreislauf-Erkrankungen belegt - ein Zusatznutzen unter Hormonentzug.
Bei der Wahl des Granatapfel-Produktes ist Vorsicht geboten, da sich die verschiedenen Produkte stark in Gehalt und Zusammensetzung der wirksamen Polyphenole unterscheiden. Durch Fermentation kann die Bioverfügbarkeit der Polyphenole erhöht werden. Im Auftrag von Natürlich gesund e.V. hat die Universität Hohenheim Gehalt und Zusammensetzung der Wirkstoffe (Polyphenole, Punicalagin) in den verschiedenen GranatapfelProdukten untersucht. GranaProstan ferment hatte von den getesteten Granatapfel-Extrakten den höchsten Punicalagin-Gehalt sowie das beste Preis-Polyphenol-Verhältnis. Den Test und einen fachlich fundierten Ratgeber können Sie auf www.granatapfelsaft. de bestellen.

Nach Informationen von

Natürlich gesund e.V., Aarbergen

\section{mRCC-Therapie: Effektivität und Verträglichkeit optimieren}

— In den letzten Jahren wurde die Therapie des metastasierten Nierenzellkarzinoms (mRCC) durch die Einführung neuer Substanzen revolutioniert. „Allerdings müssen wir den Einsatz der Substanzen optimieren in Hinblick auf aktuelle Studien. Dazu bietet das interaktive Kidney Kolleg Kompakt eine exzellente Plattform", berichtete Prof. Christian Doehn, Lübeck. Vor allem der Einstieg in die Therapie - also die First-Line-Therapie - sollte für die Patienten nicht nur so effektiv sondern auch so verträglich wie möglich sein. Dadurch könnten die Patienten dem Therapieziel, so lang wie möglich in der Behandlung gehalten zu werden, möglichst nah kommen. Als besonders effektive Therapieoption sieht Doehn in diesem Zusammenhang die Kombination aus Bevacizumab (Avastin ${ }^{\circ}$ ) und Interferon-alpha-2a (IFN). Bereits in der Zulassungsstudie AVOREN konnte die hohe Effektivität dieser Kombination unter Beweis gestellt werden [Escudier et al. 2007]. Obwohl die Therapie insgesamt gut verträglich war, ließen sich die meisten unerwünschten Wirkungen der Therapie auf das IFN zurück führen. Daher wurde nun in der multinationalen, einarmigen Phase-II-Studie BEVLiN erstmalig niedrig dosiertes IFN mit Bevacizumab eingesetzt [Melichar et al. 2011]. In der Studie erhielten 147 nicht vorbehandelte Patienten mit einem mRCC und gutem bis mittlerem Risikoscore nach Motzer-Kriterien Bevacizumab ( $10 \mathrm{mg} / \mathrm{kg}$ alle 2 Wochen) plus IFN in einer Dosierung von 3 Mio. I.E. dreimal wöchentlich. Die Resultate zeigen, dass sich IFNassoziierte Nebenwirkungen mit Grad $\geq 3$ unter der reduzierten IFN-Dosis im BEVLiNKollektiv dadurch deutlich zur AVOREN-Kontrollgruppe reduzieren ließen. Die Patienten im BEVLiN-Kollektiv erreichten ein medianes progressionsfreies Überleben (PFS) von 14,8 Monaten. Das PFS in der historischen Kon- trollgruppe betrug 10,5 Monate.„Dies ist nicht nur das längste progressionsfreie Überleben, welches jemals in einer First-Line-Studie beim mRCC erzielt wurde, sondern die Kombination eignet sich für den klinischen Alltag auch aufgrund ihres sehr guten Verträglichkeitsprofils", betonte Doehn. Bettina Reich

Meet-the-Expert „Kidney Kolleg kompakt Aktuelle Therapie des fortgeschrittenen RCC" im Rahmen des 63. DGU-Kongresses, Hamburg, 16. September 2011

Veranstalter: Roche, Grenzach-Wyhlen

\section{Spermienqualität steigern}

— Die Verbesserung der Spermienqualität und -quantität steht seit Jahrzehnten im Fokus der Wissenschaft. Vor allem acht wissenschaftlich fundierte Einzelsubstanzen ragen durch signifikante Verbesserungen einzelner Faktoren im Spermiogramm heraus: L-Carnitin, L-Arginin, Coenzym Q10, Vitamin E, Zink, Folsäure, Glutathion und Selen. Aktuelle Studien zeigen, dass jedoch erst die Kombination dieser Mikronährstoffe die Einzeleffekte synchronisiert und dadurch die Samenzelldichte um $80 \%$ steigert. Die therapeutisch effektive Konzentration und Kombination dieser Wirkstoffe in PROfertil ${ }^{\oplus}$ (als Granulat und Kapseln) wirkt sich positiv auf Anzahl, Beweglichkeit und Formung der Spermien aus.

Nach Informationen von Steiner Arzneimittel und Aristo Pharma, Berlin 